\title{
A Comparative Study on Optical Characteristics of InGaAsP QW Heterostructures of Type-I and Type-II Band Alignments
}

\author{
Garima Bhardwaj ${ }^{1}$, Sandhya K. ${ }^{2}$, Richa Dolia ${ }^{3}$, M. Abu-Samak ${ }^{4}$, Shalendra Kumar ${ }^{5}$, \\ P. A. Alvi ${ }^{6} *$ \\ ${ }^{1,3}$ Department of Electronics, Banasthali University, Banasthali-304022, Rajasthan, India \\ ${ }^{2,6}$ Department of Physics, Banasthali University, Banasthali-304022, Rajasthan, India \\ ${ }^{4}$ Department of Physics, Al-Hussein Bin Talal University, P.O. Box 20, Ma'an, Jordan \\ ${ }^{5}$ Electronic Materials \& Nanomagnetism Lab, Department of Applied Physics, Amity School of Applied Sciences, Amity
} University Haryana, Gurgaon, 122413, India

\begin{tabular}{l} 
Article Info \\
\hline Article history: \\
Received Dec 06, 2017 \\
Revised Jan 29, 2018 \\
Accepted Feb 12, 2018
\end{tabular}

\section{Keywords:}

Heterostructures

InGaAsP

Optical gain

Quantum well

\begin{abstract}
In this paper, we have configured InGaAsP QW (quantum well) heterostructures of type-I and type-II band alignments and simulated their optical characteristics by solving 6 x 6 Kohn-Luttinger Hamiltonian Matrix. According to the simulation results, the InGaAsP QW heterostructure of type-I band alignment has been found to show peak optical gain (TE mode) of the order of $\sim 3600 / \mathrm{cm}$ at the transition wavelength $1.40 \mu \mathrm{m}$; while of type-II band alignment has achieved the peak gain (TE mode) of the order of $\sim 7800 / \mathrm{cm}$ at the wavelength of $\sim 1.85 \mu \mathrm{m}$ (eye safe region). Thus, both of the heterostructures can be utilized in designing of opto-or photonic devices for the emission of radiations in NIR (near infrared region) but form the high gain point of view, the InGaAsP of type-II band alignment can be more preferred.
\end{abstract}

\section{Corresponding Author:}

P. A. Alvi,

Department of Physics, Banasthali University,

Banasthali-304022, Rajasthan, India.

Email: drpaalvi@gmail.com

\section{INTRODUCTION}

Recently, the type-I and type-II nano-heterostructures have been very popular in the area of optoelectronics because of their demand in the in the designing of opto-devices such as detectors, lasers, LEDs and other photovoltaic devices due to their proficiency of transport of photogenerated carriers in the heterostructures. Both of them have their distinguished features in different regime of wavelengths. Most of the type-I and type-II heterostructures has shown their utility in the visible region and near infrared region (NIR) [1-6]; some have been found to play their role in MIR (mid infrared region) or SWIR (shortwave infrared region) as well as in FIR (far infrared region) [7]-[11]. Recently, nitride based type-II heterostructures have also been reported for UV (ultra violet) applications. For example, Liu et al. [12] have proposed AlInN-delta-GaN quantum well ultraviolet lasers and claimed their high optical as compared to the conventional nitride heterostructures. Also, Tan et al. [13] have reported optical gain and spontaneous emission characteristics of low In-content AlInN-delta-GaN quantum wells (QWs) and analyzed the results for deep ultraviolet (UV) light emitting diodes (LEDs) and lasers. In their analysis, they found a large increase in the dominant transverse electric (TE) polarized spontaneous emission rate and optical gain. Taniyasu et al. [14] have studied AlN/GaN short-period superlattices and have reported different polarization property from AlGaN. In reference [15], the optical gain and spontaneous emission characteristics of AlInN quantum well heterostructures for deep ultraviolet emitters have been discussed. D. Rosales et al. [16] have reported an unusual temperature dependence of exciton lifetimes in arrays of GaN nanostructures grown on 
semipolar (11-22) oriented Al0.5Ga0.5N alloy by means of MBE (molecular beam epitaxy) technique. Moreover, Ngo et al. [17] have studied photo-induced non-radiative intrinsic Auger recombination processes for red, yellow and green light emitting InGaN/GaN heterostructures grown along the polar orientation.

Apart from the detailed study of type-I and type-II heterostructures, their transformations have also been studied. For example, in reference [18], P. A. Alvi has shown that the InAs/AlSb materials based heterostructure with type-II band alignment can be transformed into type-I heterostructure. Moreover, the transformed type-I heterostructure has found to exhibit improved optical gain. However, so far, the behavior of optical characteristics of any particular QW material system with type- I and type-II band alignments have not studied. Hence, in this the paper the efforts have been made for comparative study on optical gain characteristics of InGaAsP QW heterostructure with the band alignments of type-I and type-II. In order to optimize the gain characteristics of the InGaAsP QW heterostructure, k.p method has been adopted. In the following sections of the paper, device structure having type-I and type-II band lineup; detailed theory adopted for gain calculation along with the results have been discussed.

\section{DEVICE STRUCTURE AND THEORY}

For type-I InGaAsP QW heterostructure, the energy band diagram showing type-I band alignment is shown in Figure 1 and corresponding design parameters are listed in Table 1. The compositions of QW and barrier materials are selected in such a way so that the bands can be aligned of type-I in nature. The substrate is selected as InP on which the heterostructure is supposed to be grown. The reason behind the selection of InP substrate is to reduce the strain induced in the well region which occurs due to lattice mismatch. If strain induced is large, the device performance may be degraded. Refer Figure 1 and Figure 2, in case of type-I band alignment it can be observed that the conduction band offset of QW region is greater than valence band offset, see Table 1; while in case of type-II band alignment the situation is reversed i.e. valence band offset of QW region is greater than conduction band offset, see Table 2.

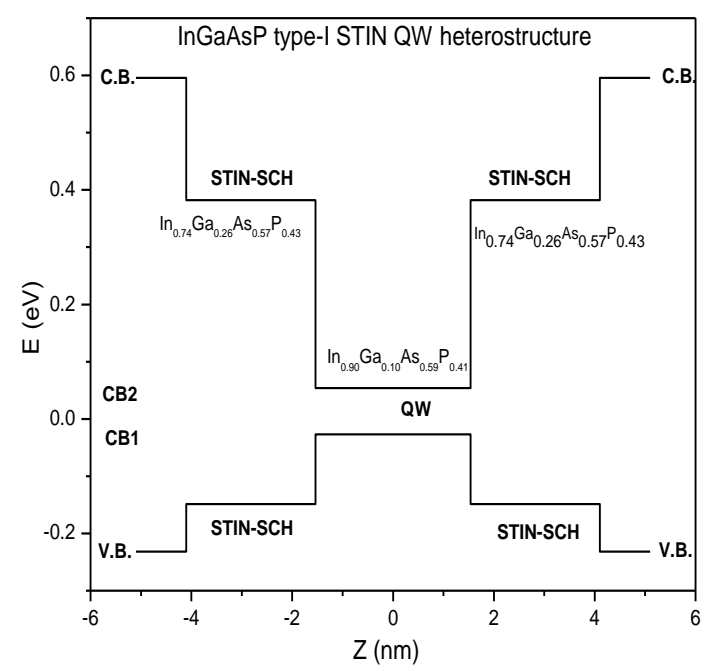

Figure 1. Energy band diagram of InGaAsP heterostructure showing type-I band alignment



Figure 2. Energy band diagram of InGaAsP heterostructure showing type-II band alignment

Table 1. Design Parameters of InGaAsP QW Heterostructure of Type-I Band Alignment

\begin{tabular}{ccccccc}
\hline $\begin{array}{c}\text { Role of } \\
\text { Layer }\end{array}$ & $\begin{array}{c}\text { Layers } \\
\text { Specification }\end{array}$ & $\begin{array}{c}\text { Energy } \\
\text { Band } \\
\text { gap } \\
(\mathrm{eV})\end{array}$ & $\begin{array}{c}\text { Lattice } \\
\text { constants } \\
(\AA)\end{array}$ & Strain & $\begin{array}{c}\text { Conduction } \\
\text { band edge- } \\
\text { offset } \\
(\mathrm{eV})\end{array}$ & $\begin{array}{c}\text { Valence } \\
\text { band } \\
\text { edge-offset } \\
(\mathrm{eV})\end{array}$ \\
\hline $\begin{array}{c}\text { Quantum } \\
\text { well }\end{array}$ & $\mathrm{In}_{0.90} \mathrm{Ga}_{0.10} \mathrm{As}_{0.59} \mathrm{P}_{0.41}$ & 0.81 & 5.93 & -0.011 & 0.048 & -0.024 \\
$\begin{array}{c}\text { Barrier } \\
\text { Cladding }\end{array}$ & $\begin{array}{c}\mathrm{In}_{0.74} \mathrm{Ga}_{0.26} \mathrm{As}_{0.57} \mathrm{P}_{0.43} \\
\mathrm{In}_{0.50} \mathrm{Ga}_{0 .} \mathrm{As}_{0.11} \mathrm{P}_{0.89}\end{array}$ & 0.98 & 5.86 & 0.013 & 0.060 & -0.094 \\
Substrate & $\mathrm{InP}$ & 1.3 & 5.94 & -0.013 & 0.176 & -0.275 \\
\hline
\end{tabular}


Table 2. Design Parameters of InGaAsP QW Heterostructure of Type-II Band Alignment

\begin{tabular}{ccccc}
\hline $\begin{array}{c}\text { S. } \\
\text { No. }\end{array}$ & $\begin{array}{c}\text { Layer } \\
\text { Specification }\end{array}$ & Role of Layer & $\begin{array}{c}\text { Conduction } \\
\text { band-offset }(\mathrm{eV})\end{array}$ & $\begin{array}{c}\text { Valence band-offset } \\
(\mathrm{eV})\end{array}$ \\
\hline 1. & $\mathrm{In}_{0.5} \mathrm{Ga}_{0.5} \mathrm{As}_{0.8} \mathrm{P}_{0.2}$ & Quantum wells & 0.190 & 0.263 \\
2. & $\mathrm{GaAs}_{0.5} \mathrm{Sb}_{0.5}$ & $\begin{array}{c}\text { Barrier } \\
\text { (Spacer) }\end{array}$ & 0.330 & 0.483 \\
3. & $\mathrm{InP}$ & Substrate & - & - \\
\hline
\end{tabular}

To determine the band structure of the heterostructures under study, k.p method has been utilized [19]. For the calculations of wavefunctions, probability distribution function (which confirm the localizations of the carriers) and discrete energy levels in valence band, the 6 x 6 Luttinger-Hamiltonian, as given below, along with effective mass approximation is solved

$$
\mathbf{H}^{L K}=-\left[\begin{array}{cccccc}
P+Q & -S & R & 0 & -S / \sqrt{2} & \sqrt{2} R \\
-S^{+} & P-Q & 0 & R & -\sqrt{2} Q & \sqrt{3} / 2 S \\
R^{+} & 0 & P-Q & S & \sqrt{3} / 2 S^{+} & \sqrt{2} Q \\
0 & R^{+} & S^{+} & P+Q & -\sqrt{2} R^{+} & -S^{+} / \sqrt{2} \\
-S^{+} / \sqrt{2} & -\sqrt{2} Q^{+} & \sqrt{3} / 2 S & -\sqrt{2} R & P+\Delta & 0 \\
\sqrt{2} R^{+} & \sqrt{3} / 2 S^{+} & \sqrt{ } 2 Q^{+} & -S / \sqrt{2} & 0 & P+\Delta
\end{array}\right]
$$

where $\Delta$ is band edge energy of the spin orbit split-off band and the superscript "+" represents the Hermetian conjugate of the parameters $\mathrm{P}, \mathrm{Q}, \mathrm{R}$ and $\mathrm{S}$. The matrix elements of the above matrix are detailed as follows:

$$
\begin{array}{cc}
P=\frac{\hbar^{2} \gamma_{1}}{2 m_{0}}\left(k_{x}^{2}+k_{y}^{2}+k_{z}^{2}\right) ; & R=\frac{\hbar^{2}}{2 m_{0}}\left[-\sqrt{3^{\gamma}} \gamma_{2}\left(k_{x}^{2}-k_{y}^{2}\right)+i 2 \sqrt{3} \gamma^{\gamma} k_{x} k_{y}\right] \\
Q=\frac{\hbar^{2} \gamma_{2}}{2 m_{0}}\left(k_{x}^{2}+k_{y}^{2}-2 k_{z}^{2}\right) ; & S=\frac{\hbar^{2} \gamma_{3}}{m_{0}} \sqrt{3}\left(k_{x}-i k_{y}\right) k_{z}
\end{array}
$$

In the above equations, $k_{x}, k_{y}$ and $k_{z}$ are the resolved components of the wave vector along $x, y$ and $z$ axis;

\begin{tabular}{|c|c|c|c|c|c|}
\hline Parameters & $\gamma_{1}$ & $\gamma_{2}$ & $\gamma_{3}$ & $\begin{array}{l}\text { Effective mass } \\
\text { of electrons }\end{array}$ & $\begin{array}{c}\text { Effective mass } \\
\text { of holes }\end{array}$ \\
\hline InAs & 20.0 & 8.50 & 9.20 & $m_{\Gamma}=0.026 m_{\mathrm{o}}$ & $\begin{array}{l}m_{h h}=0.333 m_{\mathrm{o}} \\
m_{l h}=0.027 m_{\mathrm{o}}\end{array}$ \\
\hline GaAs & 6.98 & 2.06 & 2.93 & $0.067 m_{\mathrm{o}}$ & $\begin{array}{l}m_{h h}=0.350 m_{\mathrm{o}} \\
m_{l h}=0.090 m_{\mathrm{o}}\end{array}$ \\
\hline InP & 5.05 & 1.6 & 2.1 & $m_{\Gamma}=0.0795 m_{\mathrm{o}}$ & $\begin{array}{c}m_{h h}=0.60 m_{\mathrm{o}} \\
m_{l h}=0.089 m_{\mathrm{o}}\end{array}$ \\
\hline $\mathrm{GaP}$ & 4.05 & 0.49 & 2.93 & $m_{\Gamma}=0.09 m_{\mathrm{o}}$ & $\begin{array}{l}m_{h h}=0.79 m_{\mathrm{o}} \\
m_{l h}=0.14 m_{\mathrm{o}}\end{array}$ \\
\hline
\end{tabular}
and the $\gamma_{1}, \gamma_{2}$, and $\gamma_{3}$ are the Luttinger parameters, which have been listed in Table 2.

Table 2. Parameters of Materials Used in Designing InGaAsP/GaAsSb Heterostructure [20], [21]

In order to obtain the optical properties of the heterostructure such as optical gain the precise formula is given as [21];

$$
\mathrm{G}(\hbar \omega)=\frac{2 . \pi e^{2}}{\mathrm{nc} \epsilon \omega \mathrm{Lm}^{2}} \sum_{\sigma=\mathrm{U}, \mathrm{L}, \mathrm{m}} \sum_{\mathrm{n}} \int\left|\left(\widehat{\mathrm{e}} . \mathrm{M}_{n m}^{\eta \sigma}\left(\mathrm{k}_{\mathrm{t}}\right)\right)\right|^{2} \times \frac{\left(f_{\mathrm{n}}^{\mathrm{c}}\left(\mathrm{k}_{\mathrm{t}}\right)-f_{\sigma \mathrm{m}}^{\mathrm{v}}\left(k_{t}\right)\right)\left(\frac{\gamma}{\pi}\right)}{\left(E_{\eta, \sigma n m}^{c, v}\left(k_{t}\right)-\omega \hbar\right)^{2}+\gamma^{2}} \frac{k_{t} d k_{t}}{2 \pi}
$$


where $e$ represents magnitude of elementary charge, $\mathrm{m}$ is mass of electron, $\hat{\mathrm{e}}$ represents the polarization vector of electric field responsible for optical effects, $\mathrm{L}$ is well width, $\mathrm{n}$ is refractive index of QW region, $\gamma=\hbar / \tau_{\text {int }}$ represents the half linewidth of the Lorentzian function and $f_{\mathrm{n}}^{\mathrm{c}}$ and $f_{\sigma \mathrm{m}}^{\mathrm{v}}$ are the quasi Fermi levels and can be given as;

$$
f_{n}^{c}\left(k_{t}\right)=\frac{1}{1+\exp \left(\frac{E_{n}^{c}\left(k_{t}\right)-F_{c}}{K_{B} T}\right)} \quad \text { and } \quad f_{\sigma m}^{v}\left(k_{t}\right)=\frac{1}{1+\exp \left(\frac{E_{\sigma m}^{v}\left(k_{t}\right)-F_{v}}{K_{B} T}\right)}
$$

and the quantity $M_{n m}^{\eta \sigma}\left(k_{t}\right)$ represents the momentum matrix element, can be defined as $M_{n m}^{\eta \sigma}\left(k_{t}\right)=$ $\left\langle\psi_{m, k t}^{v \sigma}|\hat{p}| \psi_{\mathrm{kt}}^{\mathrm{c \eta}}\right\rangle$, where $\hat{p}$ is the momentum operator. For TE mode, the polarization vector of electric field is taken as $\hat{\mathrm{e}}=\hat{x}$; while for TM mode, it is taken as $\hat{\mathrm{e}}=\hat{z}$. The term $E_{\eta, \sigma n m}^{c, v}\left(k_{t}\right)$, basically, represents the energy gap between the conduction $(n)$ and valence sub-bands $(m)$ and can be given as;

$$
E_{\eta, \sigma n m}^{c, v}\left(k_{t}\right)=E_{n}^{c}\left(k_{t}\right)-E_{\sigma m}^{v}\left(k_{t}\right)
$$

\section{SIMULATION RESULTS AND DISCUSSION}

The basic and very important requirement for the realization of optoelectronic devices such as LEDs and lasing heterostructures is to determine the optical gain because it describes the optical amplification in active region $(\mathrm{QW})$ the semiconductor based QW heterostructure. Actually, the gain is a process where the medium of the active region of the heterostructure transfers some of its energy to the emitted EM (electromagnetic) radiation, which results into an increased optical power. Hence, understanding gain process is a major objective as being a basic requirement for optimization of QW heterostructures. For InGaAsP material system based QW heterostructures with type-I and type-II band alignments, the optical gain has been calculated and plotted in Figure 3 and 4.

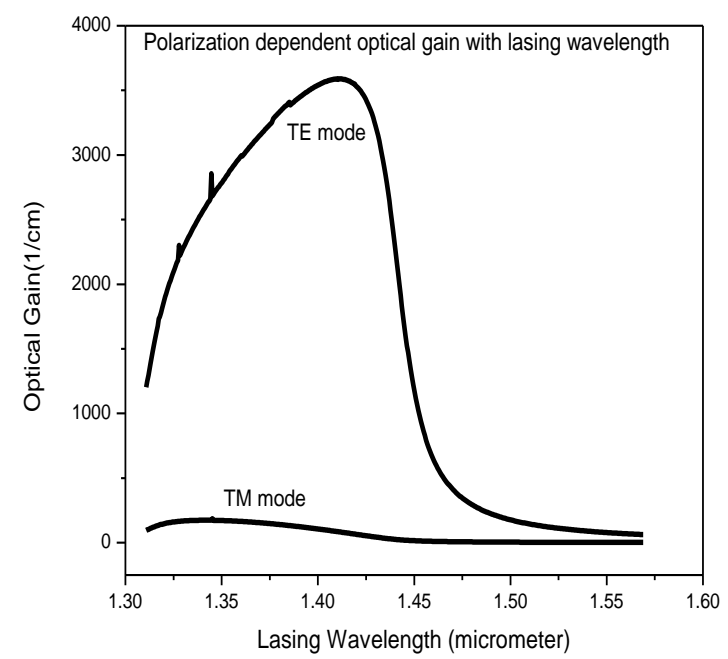

(a)

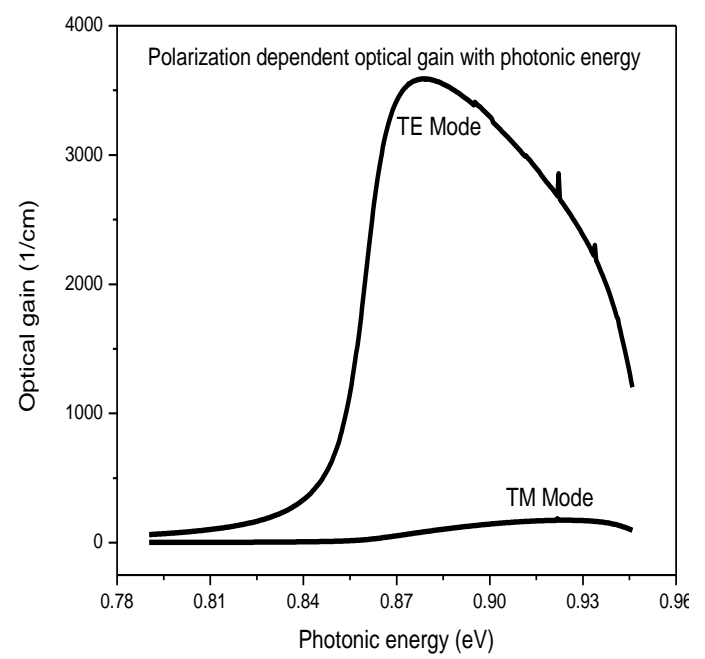

(b)

Figure 3. Polarization dependent optical gain spectra as function of (a) lasing wavelength and (b) photonic energy for InGaAsP type-I STIN QW heterostructures 


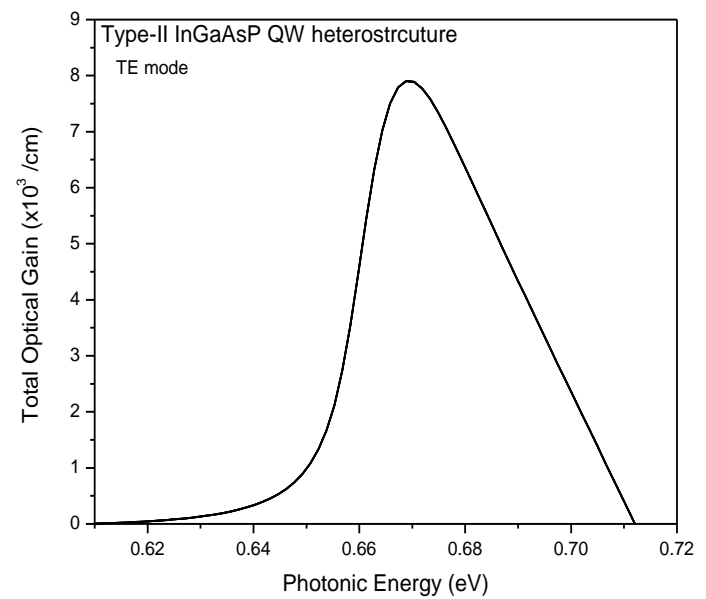

(a)

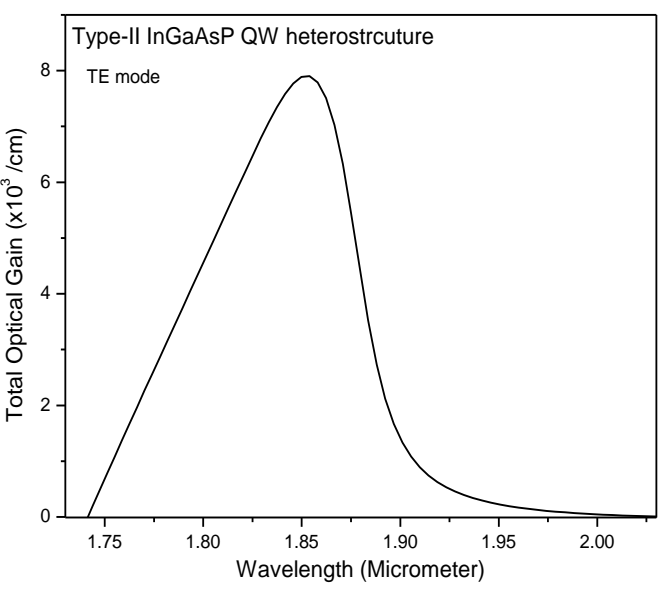

(b)

Figure 4. Behavior of total optical gain (a) in terms of energy (b) in terms of wavelength of InGaAsP typeII STIN QW heterostructure

In Figure 3, for the type-I InGaAsP based QW heterostructure grown on the InP substrate, the polarization dependent optical gain is plotted. In figure 3 (a) and (b), the optical gain (TE and TM) as a function of wavelength and photonic energy, respectively, is plotted. In these figures it can be observed that the peak optical gain with in TE mode (of the order of $\sim 3600 / \mathrm{cm}$ ) is achieved at the wavelength 1.40 $\mu \mathrm{m}$ with corresponding photonic energy $0.88 \mathrm{eV}$; while within TM mode this gain is very low (of the order of $\sim 50 / \mathrm{cm}$ ) and it occurs at wavelength $1.34 \mu \mathrm{m}$ with corresponding photonic energy $0.925 \mathrm{eV}$

Now, refer Figure 4, behavior of total optical gain in terms of energy and transition wavelength of InGaAsP STIN QW heterostructure with type-II band alignment is shown. In figure 4 (a) and (b), the optical gain (TE mode only) as a function of photonic energy and wavelength, respectively, is plotted. In these figures it can be observed that the peak optical gain with in TE mode (of the order of $7800 / \mathrm{cm}$ ) is achieved at the wavelength $1.85 \mu \mathrm{m}$ (eye safe region) with corresponding photonic energy $0.67 \mathrm{eV}$. So, keeping in views, the magnitude of optical gain only, the type-II aligned InGaAsP QW heterostructure can be preferred for the application of designing the nanoscale diode structure that emit high intense optical radiation within NIR (near infrared region). The reason behind the achievement of high optical gain in type-II InGaAsP heterostructure is that this structure has two quantum wells of InGaAsP material separated by GaAsSb spacer layer and hence dominant transitions resulting into high optical gain is achieved; while type-I aligned InGaAsP QW heterostructure has single QW, see Figure 1 and Figure 2, and therefore a less strong transition is achieved which causes low optical gain.

However, the lattice mismatching also plays a very important role in deciding the emission wavelength and optical gain, because due to lattice mismatch (that occurs due to difference in lattice constants of materials of substrate and quantum wells or barriers) a strain (compressive or strain) comes into play by which the band structure and hence band gap is changed that results into the changed wavelength. Also, the variation in strain can be seen by changing the molar composition of either substrate material or quantum well material. In reference [23], the variation in strain in nitride based heterostructures due to composition variation has been shown. Another option to enhance the optical gain and wavelength of the heterostructure is to apply the external pressure, because upon application of external pressure on the heterostructure, the band structure may be changed depending on the direction of pressure applied. For example, in figure 5, the bulk band structure of InGaAsP semiconductor compound on InP substrate is shown. Thy system has tensile strain. In this situation, light hole (green color) is found to lie above the heavy hole (green color). The existence of tensile strain confirms the lattice mismatch between the InGaAsP and InP materials. But, if the uniaxial pressure is applied the structure shown in figure 5, definitely it will be changed. 


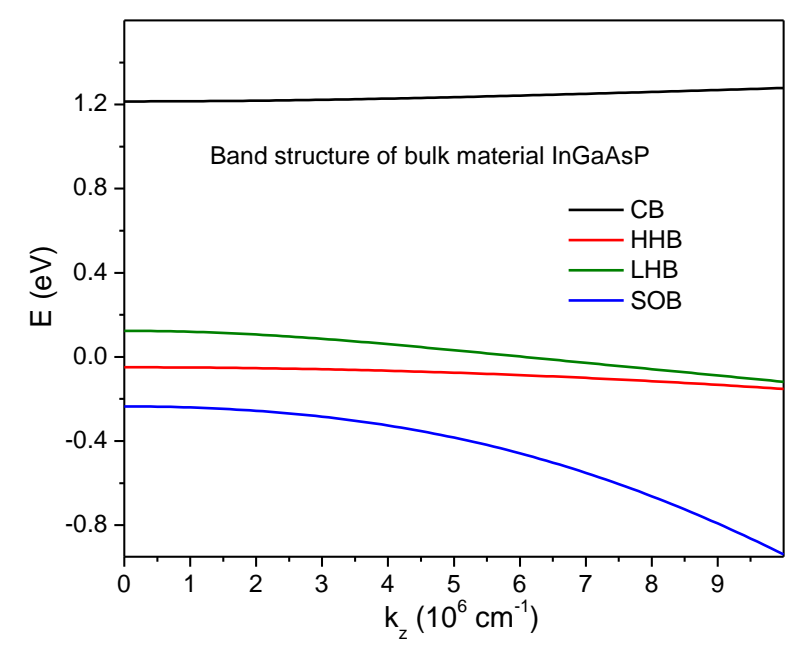

Figure 5. Bulk band structure of InGaAsP semiconductor compound on InP substrate

\section{CONCLUSION}

InGaAsP QW based heterostructures with type-I and type-II band alignments have been studied and their optical characteristics have been simulated by using k.p method. On the behalf of outcomes of the calculations carried out, the InGaAsP QW heterostructure of type-I band alignment has been found to show peak optical gain (TE mode) of the order of $\sim 3600 / \mathrm{cm}$ at the transition wavelength $1.40 \mu \mathrm{m}$; while of type-II band alignment has achieved the peak gain (TE mode) of the order of $\sim 7800 / \mathrm{cm}$ at the wavelength of $\sim 1.85$ $\mu \mathrm{m}$ (eye safe region).

\section{ACKNOWLEDGEMENTS}

P. A. Alvi, and Garima Bhardwaj are grateful to "Banasthali Center for Research and Education in Basic Sciences" ' under CURIE programme supported by the DST, Government of India, New-Delhi.

\section{REFERENCES}

[1] Pyare Lal, Shobhna Dixit, F. Rahman, P. A. Alvi, "Gain Simulation of Lasing Nano-Heterostructure $\mathrm{Al}_{0.10} \mathrm{Ga}_{0.90} \mathrm{As} / \mathrm{GaAs}$ ", Physica E: Low-dimensional systems and Nanostructures, 46, pp. 224-231 (2012).

[2] P. A. Alvi, Pyare Lal, S. Dalela, M. J. Siddiqui, "An Extensive Study on Simple and GRIN SCH based $\mathrm{In}_{0.71} \mathrm{Ga}_{0.21} \mathrm{Al}_{0.08} \mathrm{As} / \mathrm{InP}$ Lasing heterostructure", Physica Scripta, 85, 035402 (2012).

[3] Pyare Lal, Rashmi Yadav, Meha Sharma, F. Rahman, S. Dalela, P. A. Alvi, "Qualitative analysis of gain spectra of InGaAlAs/InP lasing nano-heterostructure” International Journal of Modern Physics B, Vol. 28, No. 29, pp. 1450206 (2014).

[4] M. Bajdaa, W. Trzeciakowski and J.A. Majewski, "Wide Range Wavelength Tuning of InGaAsP/InP Laser Diodes", Acta Physica Polonica A, No. 5, Vol. 120, 852-855 (2011).

[5] Hongping Zhao and Nelson Tansu, "Optical gain characteristics of staggered InGaN quantum wells lasers", J. of Appl. Phys. 107, 113110 (2010).

[6] C. Berger, C. Möller, P. Hens, C. Fuchs, W. Stolz, S. W. Koch, A. Ruiz Perez, J. Hader, and J. V. Moloney, "Novel type-II material system for laser applications in the near-infrared regime", AIP Advances, 047105 (2015).

[7] H. K. Nirmal, Nisha Yadav, F. Rahman, P. A. Alvi, "Optimization of high optical gain in type -II $\mathrm{In}_{0.70} \mathrm{Ga}_{0.30} \mathrm{As} / \mathrm{GaAs}_{0.40} \mathrm{Sb}_{0.60}$ lasing nano-heterostructure for SWIR applications”, Superlattices and Microstructures, Vol. 88, pp. 154-160 (2015).

[8] H. K. Nirmal, Nisha Yadav, S. Dalela, Amit Rathi, M. J. Siddiqui, P. A. Alvi, "Tunability of optical gain (SWIR region) in type-II $\mathrm{In}_{0.70} \mathrm{Ga}_{0.30} \mathrm{As} / \mathrm{GaAs}_{0.40} \mathrm{Sb}_{0.60}$ nano-heterostructure under high pressure", Physica E: Lowdimensional systems and Nanostructures, 80, pp. 36-42 (2016).

[9] Nisha Yadav, Garima Bhardwaj, S. G. Anjum, S. Dalela, M. J. Siddiqui, P. A. Alvi, "Investigation of high optical gain in complex type-II InGaAs/InAs/GaAsSb nano-scale heterostructure for MIR applications", Applied Optics, Vol. 56, No. 15 (2017). 
[10] Garima Bhardwaj, Nisha Yadav, S. G. Anjum, M. J. Siddiqui, P. A. Alvi, "Uniaxial strain induced optical properties of complex type-II InGaAs/InAs/GaAsSb nano-scale heterostructure", International Journal of light \& electron optics: Optik, 146 8-16 (2017).

[11] Baile Chen, W.Y. Jiang, A.L. Holmes Jr., "Design of strain compensated InGaAs/GaAsSb type-II quantum well structures for mid-infrared photodiodes”, Opt. Quantum Electron. 44 (3-5), 103-109 (2012).

[12] Cheng Liu, Yu Kee Ooi, and Jing Zhang, "Proposal and physics of AlInN-delta-GaN quantum well ultraviolet lasers", Journal of Applied Physics 119, 083102 (2016).

[13] Chee-Keong Tan, Wei Sun, Damir Borovac \& Nelson Tansu, "Large Optical Gain AlInN-Delta-GaN Quantum Well for Deep Ultraviolet Emitters", Sci. Rep. 6, 22983; doi: 10.1038/srep22983 (2016).

[14] Taniyasu, Y. \& Kasu, M. "Polarization property of deep-ultraviolet light emission from C-plane AlN/GaN shortperiod superlattices", Appl. Phys. Lett. 99, 251112 (2011).

[15] Tan, C. K. \& Tansu, N. "Gain and spontaneous emission characteristics of AlInN quantum well for deep ultraviolet emitters" Proc. of the IEEE Photonics Conference, 577-578 (2015).

[16] D. Rosales, T. Bretagnon, and B. Gil, A. Kahouli, J. Brault, B. Damilano, and J. Massies, M. V. Durnev, A. V. Kavokin, "Excitons in nitride heterostructures: From zero- to one-dimensional behavior", Phy. Rev. B, 88, 125437 (2013).

[17] Thi Huong Ngo, Bernard Gil, Benjamin Damilano, Kaddour Lekhal, Philippe De Mierry, "Internal quantum efficiency and Auger recombination in green, yellow and red InGaN-based light emitters grown along the polar direction", Superlattices and Microstructures 103, 245-251 (2017).

[18] P. A. Alvi, "Transformation of type-II InAs/AlSb nanoscale heterostructure into type-I structure and improving interband optical gain”, Phys. Status Solidi B 254, No. 5, 1600572 (2017).

[19] P. A. Alvi, Sapna Gupta, Meha Sharma, Swati Jha, F. Rahman, "Computational modeling of novel $\operatorname{InN} / \mathrm{Al}_{0.30} \mathrm{In}_{0.70} \mathrm{~N}$ multilayer nano-heterostructures", Physica E: Low-dimensional systems and Nanostructures, 44, pp. 49-55 (2011).

[20] Yoon-Suk Kim, Martijn Marsman, and Georg Kresse, "Towards efficient band structure and effective mass calculations for III-V direct band-gap semiconductors", Phys. Rev. B, 82, 205212 (2010).

[21] I. Vurgaftman, J. R. Meyer, and L. R. Ram-Mohan, "Band parameters for III-V compound semiconductors and their alloys", J. Appl. Phys. 89, 5815 (2001).

[22] Shun Lien Chuang, Physics of Photonic Devices, Willey \& Sons, Inc., $2^{\text {nd }}$ Edition (2009).

[23] Sapna Gupta, F. Rahman, M. J. Siddiqui, P. A. Alvi, "Strain Profile in III-Nitride based Multilayer Nanoheterostructures”, Physica B: Condensed Matter, Vol. 411, pp. 40-47 (2013). 